\title{
Identification of bone morphogenetic protein 4 in the saliva after the placement of fixed orthodontic appliance
}

\author{
Lovorka Grgurevic ${ }^{1,2^{*}}$ D, Ruder Novak', Grgur Salai ${ }^{1}$, Vladimir Trkulja ${ }^{3}$, Lejla Ferhatovic Hamzic ${ }^{1}$,
} Vojka Zgombic Popovic ${ }^{4}$ and Darko Bozic ${ }^{5}$

\begin{abstract}
Background: This study was conducted in order to explore the effects of orthodontic tooth movement (OTM) on the changes of salivary proteome. This prospective observational pilot study recruited 12 healthy teenage boys with malocclusion treated with a fixed orthodontic appliance and 6 appropriate control participants. Saliva samples were collected a day before and at 0,2,7, and 30 days after initialization of treatment, corresponding to the initial, lag, and post-lag phases of OTM. Pooled samples were analyzed by liquid chromatography-mass spectrometry, ELISA, and Western blotting. To date, there is no published data on the presence of BMP molecules or their antagonists in the saliva or in the gingival cervical fluid related to orthodontic conditions.

Results: A total of 198 identified saliva proteins were classified based on their functional characteristics. Proteins involved in bone remodeling were observed exclusively 30 days post appliance placement, including bone morphogenetic protein 4 (BMP4), a BMP antagonist BMP-binding endothelial regulator, insulin-like growth factorbinding protein 3, cytoskeleton-associated protein 4, and fibroblast growth factor 5. Based on the analysis of protein interactions, BMP4 was found to have a central position in this OTM-related protein network.

Conclusions: The placement of a fixed orthodontic appliance induced occurrence of proteins involved in bone remodeling in the saliva at a time corresponding to the post-lag period of OTM. Limitations of this study include a relatively small sample size, limited time of monitoring patients, and the lack of interindividual variability assessment.
\end{abstract}

Keywords: Saliva, BMP4, BMPER, Proteomics, Orthodontic tooth movement, Bone remodeling

\section{Background}

Orthodontic tooth movement (OTM) is a mechanically induced process used to treat malocclusion, a misalignment, or incorrect relation between the teeth of the two dental arches. Alveolar bone adapts to the mechanical strains caused by the placement of an orthodontic appliance that is used to correct such malocclusions [1]. In

\footnotetext{
* Correspondence: lovorka.grgurevic@mef.hr

'Center for Translational and Clinical Research, Department of Proteomics,

School of Medicine, University of Zagreb, Salata 11 Zagreb, Croatia

2Department of Anatomy, "Drago Perovic," School of Medicine, University of

Zagreb, Zagreb, Croatia

Full list of author information is available at the end of the article
}

the initial phase, tooth movement depends on the periodontal ligament (PDL), a dense and heterogeneous fibrous connective tissue structure that anchors the teeth to the alveolar bone [2]. It is the most deformable tissue in the periodontium that allows the initial mechanical disruption of the teeth. A lag phase follows when no tooth movement occurs over 4 to 20 days. In this phase, compression of the PDL causes disturbances in its blood flow, leading to cell death and hyalinization [3]. In the post-lag phase, the dead cells are resorbed by resident macrophages and bone remodeling is initiated. Applied mechanical force triggers bone resorption at the 
pressure side and bone formation at the tension side resulting in tooth movement about 40 days after the initial force application [4]. Numerous inflammatory mediators, cytokines, growth factors, and bone remodelingrelated proteins are expressed during OTM; however, the exact mechanism of osteogenesis on the tension side is poorly understood [5].

The salivary proteome has a prominent serum component that is predominantly sourced from the blood vessels of the carotid artery. Most compounds found in saliva are also found in the blood, so changes in the protein composition of the saliva are an indicator of not only local, but also systemic changes that can be associated with certain diseases. One of the major hurdles to the use of the saliva as a diagnostic fluid is its notorious variability in composition: influenced by sex, age, and numerous physiological processes such as pregnancy, exercise and physical activity, cigarette smoking, and stress, as well as inter-individual variation [6, 7]. Salivary proteomics during OTM has been recently reviewed suggesting that several biomarkers could be a useful tool in indicating the effectiveness of orthodontic treatment. Proposed candidates include myeloperoxidase, leptin, and the ratio of nuclear factor kappa $\mathrm{B}$ ligand to osteoprotegerin; however, their potential clinical use is overshadowed by inter-individual variation [8]. In terms of novel biomarker discovery by mass-spectrometry-based proteomics, a small number of studies have revealed only a handful of potential markers that could be linked biologically to OTM. Such example is the upregulation of apolipoprotein E, a novel regulator of bone metabolism in mice, induced by orthodontic treatment [9].

Inflammation plays a key role in the remodeling process on the compression side where PDL fibroblasts synthesize proinflammatory cytokines, which stimulate degradation of extracellular matrix and promote cell growth [10]. This aseptic inflammation is essential for highly complex mechanisms of OTM involving transforming growth factor-beta (TGFß) and bone morphogenetic proteins (BMPs) [11]. BMPs belong to the TGF $\beta$ superfamily of cytokines with pleiotropic functions in fetal and postnatal development [12]. They were first identified by their ability to induce ectopic bone and cartilage formation, but to date, no BMP molecules have been identified in the saliva. In OTM, it has recently been proposed that the PDL osteocytes, cells responsible for mechanosensing, control alveolar bone remodeling via sclerostin, a potent BMP antagonist [13]. Vascular endothelial cells present in the alveolar bone recruit circulating inflammatory cells to the PDL. This stressinduced inflammatory response is modulated by BMP signaling [14]. Once upregulated, BMP2 and BMP4 induce proinflammatory endothelial phenotype, enhancing leukocyte adhesion to the endothelial surface in vitro
$[15,16]$. Moreover, mechanical stress induces osteoblast differentiation and an increase in BMP4 gene expression in mouse preosteoblasts and fibroblasts, which suggests that BMP4 could be an important autocrine and paracrine factor in tensile stress-induced osteogenesis [17].

The proteomics-based pilot study was conducted, with intention to screen the potential short-term evaluation (over 30 days) of the human saliva proteome related to the OTM induced by the placement of the orthodontic appliance to treat malocclusion in teenage. Considering their role in the processes encompassing OTM and during tooth root and periodontium formation, it was hypothesized that members of the TGF $\beta$ superfamily of cytokines might be detected.

\section{Materials and methods Study participants}

This prospective observational pilot study included 18 consecutive patients with malocclusion, admitted at the same orthodontic clinic. The participants met the following criteria: male sex, age of 12 to 14 years, systemically healthy, non-smoker, and indication for MBT orthodontic therapy. Exclusion criteria were local or systemic disease development during the follow-up period, failure to keep follow-up appointments and patients who willingly drop-out from the study; however, no patients were excluded during this pilot study. Male patients with a similar age were chosen in order to minimize the sources of variability and bias of this pilot study. Participants' individual malocclusion types are presented in the Supplementary table S1. At the beginning of the study, the participants with malocclusions were divided into two groups, 12 cases who underwent orthodontic therapy and 6 controls who did not, for which the Straight wire MBT technique, with a slot 22 and a 0.12 nickel-titanium wire was utilized. For all participants, clinical periodontal parameters were examined by determining the pocket probing depth (PPD), full-mouth plaque score (FMPS), and full-mouth bleeding score (FMPS) $[18,19]$. These parameters were measured at six sites per tooth (mesio-buccal, buccal, disto-buccal, mesio-oral, oral, disto-oral aspects) using the UNC 15 $\mathrm{mm}$ periodontal probe (Devemed $\mathrm{GmbH}$, Tuttlingen, Germany) and are presented in Table 1. Seven days

Table 1 Participants' clinical periodontal parameters: SD standard deviation; PPD - pocket probing depth; FMPS - full mouth plaque score, FMBS - full-mouth bleeding score

\begin{tabular}{lll}
\hline Variable mean \pm SD & Cases & Controls \\
\hline PPD $(\mathrm{mm})$ & $2,30 \pm 0.2$ & $2.32 \pm 0.3$ \\
FMPS (\%) & $16 \pm 3.4$ & $15 \pm 3.8$ \\
FMBS (\%) & $14 \pm 3.6$ & $13 \pm 3.7$ \\
\hline
\end{tabular}


prior to the commencement of orthodontic therapy, all participants underwent ultrasonic supragingival instrumentation with fine tips only (EMS; Nyon, Switzerland) in order to eliminate gingival inflammation. The study was approved by the Ethics Committee at the School of Dental Medicine (05-PA-15-4/ 2017). Written informed consent was obtained from all participants and their parents. Study design is graphically depicted in Fig. 1.

\section{Saliva sampling}

Cases' saliva samples were collected 1 day prior to and then at $0,2,7$, and 30 days post orthodontic appliance placement corresponding to the initial ( 0,2 days), lag (7 days), and post-lag (30 days) phases of OTM. At the same time points, saliva samples were also collected from the 6 controls with malocclusions. Participants refrained from eating and washed their teeth $60 \mathrm{~min}$ before saliva sampling. The saliva was expectorated into clean Petri dishes, of which $1 \mathrm{~mL}$ was transferred to Eppendorf tubes and immediately frozen at $-80^{\circ} \mathrm{C}$. For analysis, all individual samples in each group, collected at one time-point were pooled, centrifuged for $10 \mathrm{~min}$ at $12000 \mathrm{~g}$ at $4^{\circ} \mathrm{C}$ to remove debris, and proteins were purified by acetone precipitation. Protein concentration was determined using RC DC Protein Assay Kit II (BioRad).

\section{Mass spectrometry}

Pooled protein samples $(40 \mu \mathrm{g})$ were alkylated $(55 \mathrm{mM}$ iodoacetamide in $8 \mathrm{M}$ urea) and digested with $0.8 \mu \mathrm{g}$ trypsin (Worthington, TPCK treated) in $10-\mathrm{kDa}$ centrifugal filter units. Digested peptides were purified on a pre-column and separated on a C18 nano-column by HPLC (Ultimate 3000, Thermo Fischer Scientific). Mass spectrometry (MS) was performed on an LTQ Orbitrap Discovery instrument (Thermo Fischer Scientific), and raw data was processed using MaxQuant software version 1.5.1.2. (Max Planck Institute of Biochemistry). Proteins were quantified using the intensity-based absolute quantification (iBAQ) algorithm with the false discovery rate at the peptide and protein level set at $1 \%$. Samples were analyzed in technical triplicates, and proteins identified with at least one peptide were considered relevant for analysis [20]. Detected proteins were classified according to their gene ontology, using the European Molecular Biology Laboratory European Bioinformatics Institute (EMBL-EBI) Quick GO web-based browser [21]. The MS proteomics data were deposited at the ProteomeXchange Consortium via the PRIDE partner repository with the dataset identifier PXD016481. STITCH database version 5.0 (http://stitch. embl.de) was used to analyze interaction networks of proteins functionally classified as bone remodeling proteins (see Supplementary methods 1 for details).

\section{BMP4 ELISA}

Pooled saliva samples obtained 30 days after placement of a fixed orthodontic appliance were applied twice to a heparin Sepharose column (Amersham Pharmacia Biotech). Eluted fractions were precipitated with saturated ammonium sulphate (SAS), and the precipitates were resuspended in phosphate-buffered saline (PBS) [22].

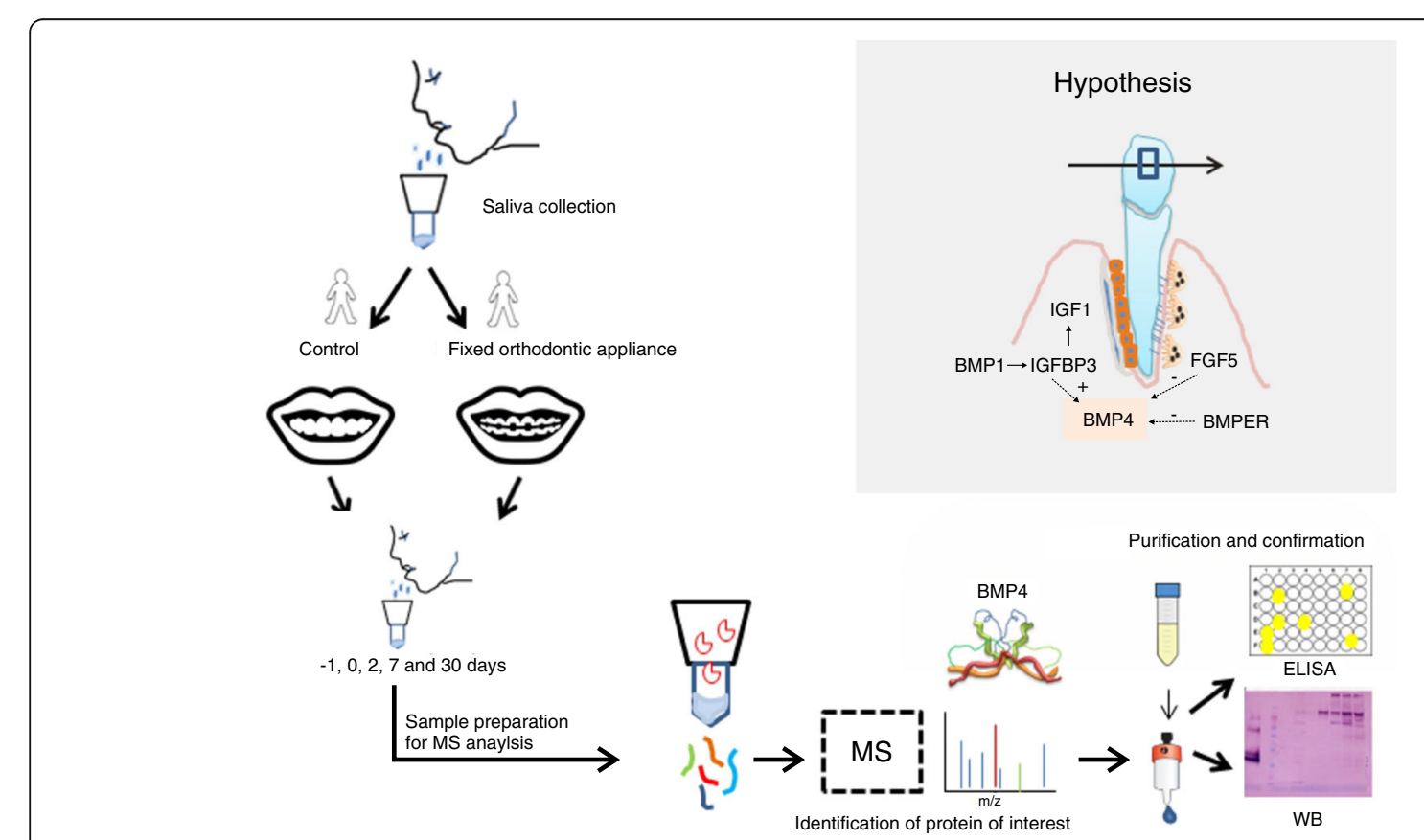

Fig. 1 Graphical summary of the conducted pilot study, including the presented hypothesis 
BMP4 protein concentration in wash and eluate solutions was measured in duplicates using BMP4 ELISA DuoSet (\#DY314, R\&D Systems) according to manufacturers' instructions (see Supplementary methods 2 for details).

\section{BMP4 Western blotting}

Heparin-enriched proteins from saliva pools were separated by SDS-PAGE and analyzed by Western blotting. rhBMP4 was detected using a mouse monoclonal antihuman BMP4 antibody, available as the capture antibody in the ELISA DuoSet kit (cat. no.\#DY314, R\&D Systems) (see Supplementary methods 3 for details).

\section{Results}

All participants were Caucasian and of European descent, which was not regulated by the inclusion/exclusion criteria. A total of 198 proteins were identified across all saliva samples (please see Supplementary Table S2 and Supplementary figure S1), which is in accordance with similar studies on the saliva [23]. They were classified into several functional subsets (Fig. 2A), but a subset of "bone remodeling-related" proteins was observed only in samples taken at 30 days post-placement of the appliance (Fig. 2A). The sample included 6 differential proteins (Table 2) with BMP4 depicted as a central protein in the interaction network (Fig. 2B). ELISA analysis suggested a detectable level of BMP4: $9.69 \mathrm{pg} / \mathrm{mL}$ in the purified/enriched samples corresponding to $0.32 \mathrm{pg} / \mathrm{mL}$ in unenriched, raw samples. Western blot (using purified, enriched samples) indicated a gentle visible band MW between 25 and $39 \mathrm{kDa}$ in the saliva 30 days following the placement of fixed orthodontic appliance (as opposed to samples taken prior to and 7 days after the placement) which could correspond to BMP4 (see Supplementary methods 3 ).

\section{Discussion}

Periodontal tissues respond to OTM with a distinct pattern. Upon placement of the fixed orthodontic appliance, mechanical disruption of the periodontal ligament allows for some tooth movement. However, a lag phase follows

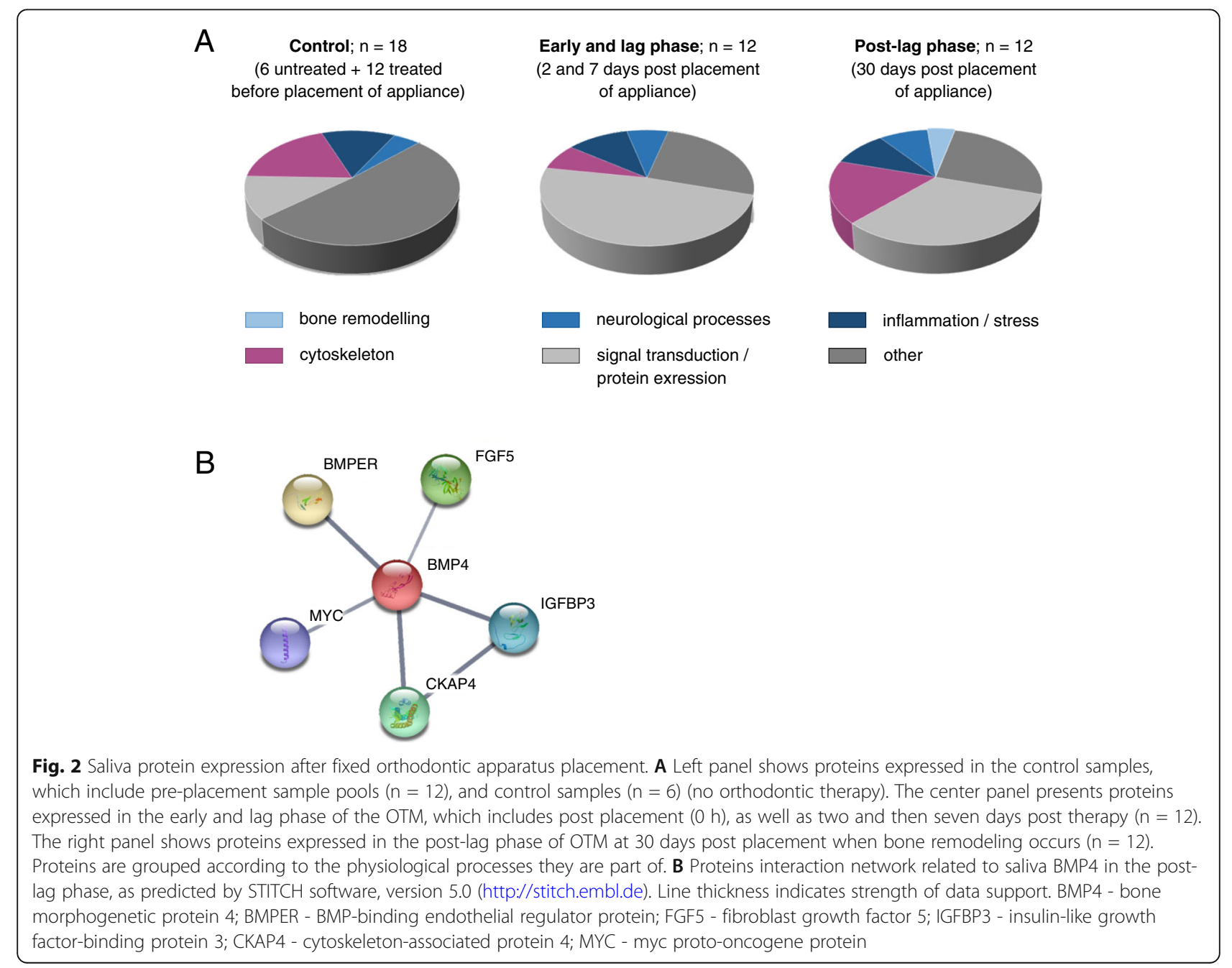


Table 2 Protein interactions identified by STICH version 5.0 related to bone morphogenetic protein 4

\begin{tabular}{llll}
\hline Protein name & Peptide sequence & UniProt ID & Gene ID \\
\hline Bone morphogenetic protein 4 & RRPQPSK & H0YMP9 & BMP4 \\
BMP-binding endothelial regulator protein & VKLRAHR & Q8N8U9 & BMPER \\
Fibroblast growth factor 5 & IPLSAPR & P12034 & FGF5 \\
Insulin-like growth factor-binding protein 3 & HRLAAGR & C9JMX4 & IGFBP3 \\
Cytoskeleton-associated protein 4 & LFVKVEK & Q07065 & CKAP4 \\
Myc proto-oncogene protein & QRRNELK & H0YBT0 & MYC \\
\hline
\end{tabular}

in which the strained tissues get inflamed and no movement occur for several weeks. Finally, as the inflammation subsides, active bone remodeling takes over through expression of specific molecules involved in periodontal regeneration and homeostasis $[1,24]$. Understanding the molecular mechanisms of OTM will advance the development of better appliances that maximize effect and minimize tissue damage. BMPs are major regulators of bone formation, and BMP4 expression in the saliva 30 days after orthodontic appliance placement indicates its possible role in alveolar bone formation and maintaining tissue homeostasis during dental displacement. Saliva BMP4 expression was confirmed by ELISA in a pooled and heparin-enriched sample 30 days after fixed orthodontic appliance placement.

The activity of BMPs is strongly influenced by developmental modulators like chordin, which prevents their activation [25]. The expression of BMPER or CV2, a chordin paralog with a clear inhibitory effect on BMP molecules, has also been confirmed for the first time during OTM in this study [26]. Recent studies show that BMPER can have antagonistic or agonistic effect on BMP molecules, depending on the relative concentration of BMPER and its ligands [26]. BMPER is expressed in the dental epithelium during development [27] where it promotes blood vessel formation by downregulation of antiangiogenic factor trombospondin-1 and upregulation of FGF2 and FGF receptor-1, potent angiogenesis promotors [28]. FGF2 is the most studied growth factor of the FGF family that is expressed in many tissues and body fluids, including the tears and saliva [29]. It induces periodontal regeneration and suppresses inflammation indicating its role in regeneration and healing [30]. In this study, FGF5 was also identified, which has the same effect on growth/differentiation balance of PDL cells, and is expressed in large amounts in PDL cell culture [31]. It has also been proposed as a regulator of BMP4 since FGF5 overexpression leads to decrease in BMP4 mRNA [32].

In OTM, depending upon its concentration, BMPER could inhibit or enhance new bone formation induced by BMP4. Additionally, it could affect the dental environment by inducing angiogenesis through the regulation of FGF molecules already identified in the saliva. Since both proteins have been identified in the saliva at the same time point, these results confirm a possible established BMP4/BMPER axis and shed new light on its role in bone remodeling during orthodontic therapy.

Another important bone metabolic pathway identified in this study is related to insulin growth factor-1 (IGF1). IGF1 induces mesenchymal to osteoblast differentiation and is highly expressed in bone matrix [33]. It is exclusively produced in the liver, from where it shortly circulates in the blood [34], and then rapidly binds to insulinlike growth factor-binding proteins (IGFBPs). IGFBP3 is the most abundant IGFBP that controls the distribution and activity of IGF-1 through interaction with its receptors. IGFBP3 also binds to type I collagen and thus plays a role in the storage of IGF1 in the bone matrix. IGFBPs are processed by the BMP1 metalloproteinase, which also releases BMP2 and BMP4 from their latent complexes. This processing also enhances some IGFindependent functions of IGFBP3 [35], including its inhibition of osteoblast differentiation through BMP2 signaling pathway suppression.

IGFBP3 is thus weaved into bone remodeling molecular networks arising during OTM. Our detection of IGFBP3 at day 30 of OTM could be related to the repair of the resorption lacunae during alveolar bone remodeling and root cementum repair. Saggese found a five-fold increase of IGF1 and IGFBP3 expression in the GCF of young patients from 8 to 15 years 1 month following orthodontic tooth movement [36].

BMP4 appears to be an important regulator of odontogenesis and tooth homeostasis. In tooth development, BMP4 is expressed in the dental follicle where it plays an important role in early tooth, alveolar bone, and soft tissue development [37]. Its expression levels remain high in postnatal bone remodeling, maturation, and homeostasis [12, 38, 39]. Increases in BMP4 expression occur in bone fractures, but importantly also during mechanical stress, and strain [17, 40]. Recent studies have found a specific mutation of the Bmp4 gene in patients with dental agenesis, which may be associated with the early onset of osteoporosis and osteopenia in humans [41]. Furthermore, BMP4 conditional knockout (BMP-4cKO) mice have a complete downregulation of dentin matrix acidic phosphoprotein 1 (DMP1) mRNA 
in cementoblasts, which is crucial for proper bone and dentin mineralization, and whose absence leads to periodontal breakdown and increased susceptibility to bacterial infection [42, 43].

BMP4 induced increased expression of cytoskeletonassociated proteins in the pluripotent stem cells [44] and, in vivo, showed odontogenic potential by inducing complete tooth formation from embryonic stem cells [45].

To the best of our knowledge, a BMP molecule was, up to now, never detected in the saliva, which raises important new questions. What is the exact role of and mechanism of action of BMP4 in alveolar bone remodeling? It also remains to be elucidated if the detected BMP4 stems locally from the GCF or does it originate from the blood, sourced from the carotid artery.

This pilot proteomic study has three main limitations. A relatively small sample size potentially does not fully represent the OTM proteome of the saliva. Therefore, only prominent bone remodeling regulators might have been identified, while weaker, but possibly important modulators might have been omitted. The second limitation refers to the time points of the study, since prolonged monitoring of the OTM saliva proteome over several months might accentuate or abate the seen effects. The third limitation is analyzing pooled samples and the lack of interindividual variability assessment: namely, saliva samples taken from boys treated with a fixed orthodontic appliance (as well as those of the control, non-treated boys) at different time points were pooled by the time-point, hence inter-individual variability of the proteomic signals could not be quantified. Considering the nature of BMPs, it is expected that their levels in small volumes of biological fluids (in this case saliva) are below the detection thresholds, so this step was practically unavoidable. However, this was a pilot study based on the "shotgun" approach, a powerful method of context-dependent signaling mechanism discovery [46]; a number of sources of variability in proteomic saliva analysis (sex, age, oral hygiene, childrennon-smokers) were controlled for by inclusion criteria; all samples were processed in the same way; and finally, control, preplacement, and early post-placement samples returned practically no signal for BMP4 and the entire cluster of "bone remodeling proteins" theoretically indicating an infinite difference between the "later lag" and "preplacement \& early lag" (i.e., between "some" vs. "none"). Finally, the presence of the molecule placed into the center of the "bone remodeling network" (BMP4) was confirmed by Western blot and ELISA. Under these circumstances, the measured concentration and the fact that purification of the samples were needed in order to establish technical pre-requisites for immunological identification of the protein are of secondary and only illustrative relevance: the fact that it could be quantified offers additional proof that this finding is not merely an artifact. Hence, despite these limitations, we believe that the present data accurately identify a biological phenomenon and highlight the need for further research into the role of BMP molecules in tooth physiology. Additionally, inherent to the study design with only boys included (purposely, to exclude gender as a source of variability and bias in this pilot study), no potential gender differences could be observed, which should be addressed in further research which will include a larger number of both male and female participants and assess interindividual variability, which will then also be correlated with periodontal measurements and examinations.

The majority of OTM occurs in the post-lag phase as a direct result of bone remodeling due to mechanical pressure of the fixed orthodontic apparatus. The need to simultaneously produce and dissolve bone tissue at opposite sides of a tooth, requires strict spatial and temporal regulation, possibly mediated by the BMP family proteins (Fig. 1). This research indicates that mechanical force could initiate a cascade of events similar to embryonic tooth development. As BMP molecules are produced locally in small amounts (on demand), they are difficult to identify in biological fluids. Mechanical forces generated during OTM have perhaps allowed us to overcome its detection level in the saliva. BMP4 and its protein networks are thus emerging as important players whose role should be further studied in bone remodeling during OTM and in homeostasis.

\section{Conclusions}

Being aware of the discussed limitations, we consider it justified to state that the present proteomic pilot study is the first to identify BMP4 protein, BMP antagonist BMP binding endothelial regulator (BMPER) or cossveinless-2 (CV2), and several other growth factors related to bone metabolism in the human saliva during the post-lag phase of OTM induced by placement of orthodontic appliances. To date, there is no published data on the presence of BMP molecules or their antagonists in the saliva or the gingival cervical fluid (GCF), regardless of different orthodontic treatments or diseases.

\section{Abbreviations}

BMP: Bone morphogenetic protein; BMP-4CKO: Bone morphogenetic protein 4 conditional knockout; BMPER: BMP-binding endothelial regulator;

CKAP: Cytoskeleton-associated protein; CV: Crossveinless; DMP: Dentin matrix acidic phosphoprotein; ELISA: Enzyme-linked immunosorbent assay; FGF: Fibroblast growth factor; MS: Mass spectrometry; GCF: Gingival cervical fluid; HPLC: High performance liquid chromatography; IGF-1: Insulin-like growth factor-1; IGFBP: Insulin-like growth factor-binding protein;

iBAQ: Intensity-based absolute quantification; LC-MS: Liquid chromatography-mass spectrometry; MYC: Myc proto-oncogene protein; OTM: Orthodontic tooth movement; PBS: Phosphate-buffered saline; PDL: Periodontal ligament; RT: Room temperature; SAS: Saturated ammonium sulphate; TGF- $B$ : Transforming growth factor $B$ 


\section{Supplementary Information}

The online version contains supplementary material available at https://doi. org/10.1186/s40510-021-00364-6

Additional file 1: Supplementary figure S1. Venn diagram of salivary proteins expressed in pooled control and orthodontic tooth movement (OTM) samples. Identification of bone morphogenetic protein 4 in saliva after placement of fixed orthodontic appliance

Additional file 2: Supplementary Methods 1. Liquid chromatography and mass spectrometry.

Additional file 3: Supplementary Methods 2. HiTrap Heparin HP column purification and BMP4 ELISA

Additional file 4: Supplementary Methods 3. SDS-PAGE and Western blot analysis of BMP4.

Additional file 5: Supplementary table S1. Participants' malocclusion types. In Class 1 malocclusion, the bite is normal, but the upper teeth slightly overlap the lower teeth. Class 2 malocclusion (retrognathism or overbite), occurs when the upper jaw and teeth severely overlap the bottom jaw and teeth. It is subdivided into division 1 where the incisors are proclined and division 2 where the incisors are retroclined

Additional file 6: Supplementary Table S2. Proteins identified across all saliva samples. Time points of control (C) and expreimental (E) sample pools are labeled $-1,0,2,7$ and 30 denoting sampling times a day before, immediately after, and then 2, 7 and 30 days after the placement of the fixed orthodontic apparatus. BR - bone remodelling, NP neurological processes, I/SR - inflammation / stress response, C cytoskeleton, ST/PE - signal transduction / protein expression, $\mathrm{O}$ - other, $U$ - unknown function.

\section{Acknowledgements}

We thank our young patients for participating in this study.

\section{Authors' contributions}

Lovorka Grgurevic, Rudjer Novak, Grgur Salai, Vladimir Trkulja, Lejla Ferhatovic Hamzic, Vojka Zgombic Popovic, and Darko Bozic all made substantial contributions to the conception or design of the work and the acquisition, analysis, revision, or interpretation of data for the work. Lovorka Grgurevic: conceptualization, formal analysis, writing —original draft, investigation supervision; Rudjer Novak: investigation, formal analysis, data curation, writing —original draft, project administration; Grgur Salai: formal analysis, investigation, writing_original draft; Vladimir Trkulja: writing—review \& editing, data curation; Lejla Ferhatović Hamzic: methodology, formal analysis, writing — review \& editing; Vojka Zgombic Popovic: methodology, investigation, writing — review \& editing; Darko Bozic: writing-review \& editing, data curation. They all revisited work critically for important intellectual content, gave final approval of the version to be published, and agreed to be accountable for all aspects of the work in ensuring that questions related to the accuracy or integrity of any part of the work are appropriately investigated and resolved. The authors read and approved the final manuscript.

\section{Funding}

This work was funded by the Scientific Center of Excellence for Reproductive and Regenerative Medicine (project "Reproductive and regenerative medicine - exploration of new platforms and potentials", Grant Agreement KK.01.1.1.01.0008 which is funded by the European Union through the European Regional Development Fund.

\section{Availability of data and materials}

The datasets supporting the conclusions of this article are available in the ProteomeXchange Consortium via the PRIDE partner repository, PXD016481, http://proteomecentral.proteomexchange.org and additional datasets are included within the article (and its additional files).

\section{Declarations}

\section{Ethics approval and consent to participate}

The study was approved by the Ethics Committee at the School of Dental Medicine, University of Zagreb (05-PA-15-4/2017). Written informed consent was obtained from all participants and their parents.

\section{Consent for publication}

Not applicable.

\section{Competing interests}

The authors declare that they have no competing interests.

\section{Author details}

${ }^{1}$ Center for Translational and Clinical Research, Department of Proteomics, School of Medicine, University of Zagreb, Salata 11 Zagreb, Croatia.

2Department of Anatomy, "Drago Perovic," School of Medicine, University of Zagreb, Zagreb, Croatia. ${ }^{3}$ Department of Pharmacology, School of Medicine, University of Zagreb, Zagreb, Croatia. ${ }^{4}$ Orthonova Dental Polyclinic, Zagreb, Croatia. ${ }^{5}$ Department of Periodontology, University of Zagreb, School of Dental Medicine, Zagreb, Croatia.

Received: 1 March 2021 Accepted: 21 May 2021

Published online: 12 July 2021

\section{References}

1. Asiry MA. Biological aspects of orthodontic tooth movement: a review of literature. Saudi J Biol Sci. 2018;25(6):1027-32 Available from: https:// pubmed.ncbi.nlm.nih.gov/30174498.

2. Li Y, Jacox LA, Little SH, Ko C-C. Orthodontic tooth movement: the biology and clinical implications. Kaohsiung J Med Sci. 2018;34(4):207-14. https:// doi.org/10.1016/j.kjms.2018.01.007.

3. von Böhl M, Kuijpers-Jagtman AM. Hyalinization during orthodontic tooth movement: a systematic review on tissue reactions. Eur J Orthod. 2009;31(1): 30-6. https://doi.org/10.1093/ejo/cjn080.

4. Reddy RS, Singaraju GS, Mandava P, Ganugapanta VR. Biology of tooth movement. Ann Essences Dent. 2015:7(4):c-21c.

5. Baloul SS. Osteoclastogenesis and osteogenesis during tooth movement. Front Oral Biol. 2016;18:75-9. https://doi.org/10.1159/000351901.

6. Murr A, Pink C, Hammer E, Michalik S, Dhople V, Holtfreter B, et al. Crosssectional association of salivary proteins with age, sex, body mass index, smoking, and education. J Proteome Res. 2017;16(6):2273-81. https://doi. org/10.1021/acs.jproteome.7b00133.

7. Marvin RK, Saepoo MB, Ye S, White DB, Liu R, Hensley K, et al. Salivary protein changes in response to acute stress in medical residents performing advanced clinical simulations: a pilot proteomics study. Biomarkers. 2017; 22(3-4):372-82 Available from: https://europepmc.org/articles/PMC5551674.

8. Allen R, Edelmann A, Abdulmajeed A, Bencharit S. Salivary protein biomarkers associated with orthodontic tooth movement: a systematic review. Orthod Craniofac Res. 2019;22(S1):14-20. https://doi.org/10.1111/ ocr.12258.

9. Zhang J, Zhou S, Zheng H, Zhou Y, Chen F, Lin J. Magnetic bead-based salivary peptidome profiling analysis during orthodontic treatment durations. Biochem Biophys Res Commun. 2012;421(4):844-9. https://doi. org/10.1016/j.bbrc.2012.04.100.

10. Davidovitch Z. Cell biology associated with orthodontic tooth movement. In: The Periodontal Ligament in Health and Disease [Internet]; 1995. p. 25978. https://doi.org/10.1093/ejo/18.6.670.

11. Krishnan V, Davidovitch Z. On a path to unfolding the biological mechanisms of orthodontic tooth movement. J Dent Res. 2009;88(7):597608. https://doi.org/10.1177/0022034509338914.

12. Pregizer SK, Mortlock DP. Dynamics and cellular localization of Bmp2, Bmp4, and Noggin transcription in the postnatal mouse skeleton. J bone Miner Res Off J Am Soc Bone Miner Res. 2015;30(1):64-70. https://doi.org/10.1002/ jbmr.2313.

13. Odagaki N, Ishihara $Y$, Wang Z, Ei Hsu Hlaing E, Nakamura M, Hoshijima M, et al. Role of osteocyte-PDL crosstalk in tooth movement via SOST/ sclerostin. J Dent Res. 2018;97(12):1374-82. https://doi.org/10.1177/0022034 518771331

14. Yao Y, Bennett BJ, Wang X, Rosenfeld ME, Giachelli C, Lusis AJ, et al. Inhibition of bone morphogenetic proteins protects against atherosclerosis 
and vascular calcification. Circ Res. 2010;107(4):485-94. https://doi.org/10.11 61/CIRCRESAHA.110.219071.

15. Helbing T, Rothweiler R, Ketterer E, Goetz L, Heinke J, Grundmann S, et al. BMP activity controlled by BMPER regulates the proinflammatory phenotype of endothelium. Blood. 2011;118(18):5040-9. https://doi.org/10.1182/blood-2 011-03-339762.

16. Vukicevic S, Grgurevic L. In: Parnham M, editor. Bone morphogenetic proteins in inflammation BT - encyclopedia of inflammatory diseases. Basel: Springer Basel; 2015. p. 1-15. https://doi.org/10.1007/978-3-0348-06206_212-1.

17. Ikegame M, Ishibashi O, Yoshizawa T, Shimomura J, Komori T, Ozawa H, et al. Tensile stress induces bone morphogenetic protein 4 in preosteoblastic and fibroblastic cells, which later differentiate into osteoblasts leading to osteogenesis in the mouse calvariae in organ culture. J Bone Miner Res Off J Am Soc Bone Miner Res. 2001;16(1):24-32. https:// doi.org/10.1359/jbmr.2001.16.1.24.

18. Ainamo J, Bay I. Problems and proposals for recording gingivitis and plaque. Int Dent J. 1975;25(4):229-35 Available from: http://europepmc.org/abstract/ MED/1058834

19. O'Leary TJ, Drake RB, Naylor JE. The plaque control record. J Periodontol. 1972;43(1):38. https://doi.org/10.1902/jop.1972.43.1.38.

20. Gupta N, Pevzner PA. False discovery rates of protein identifications: a strike against the two-peptide rule. J Proteome Res. 2009;8(9):4173-81. https://doi. org/10.1021/pr9004794.

21. Zahn-Zabal M, Michel P-A, Gateau A, Nikitin F, Schaeffer M, Audot E, et al. The neXtProt knowledgebase in 2020: data, tools and usability improvements. Nucleic Acids Res. 2020;48(D1):D328-34. https://doi.org/10.1 093/nar/gkz995.

22. Grgurevic L, Macek B, Durdevic D, Vukicevic S. Detection of bone and cartilage-related proteins in plasma of patients with a bone fracture using liquid chromatography-mass spectrometry. Int Orthop. 2007;31(6):743-51. https://doi.org/10.1007/s00264-007-0404-z.

23. Schulz BL, Cooper-White J, Punyadeera CK. Saliva proteome research: current status and future outlook. Crit Rev Biotechnol. 2013;33(3):246-59. https://doi.org/10.3109/07388551.2012.687361.

24. Ellias MF, Zainal Ariffin SH, Karsani SA, Abdul Rahman M, Senafi S, Megat Abdul Wahab R. Proteomic analysis of saliva identifies potential biomarkers for orthodontic tooth movement. Sci World J. 2012;2012:647240. https://doi. org/10.1100/2012/647240.

25. Larraín J, Bachiller D, Lu B, Agius E, Piccolo S, De Robertis EM. BMP-binding modules in chordin: a model for signalling regulation in the extracellular space. Development. 2000;127(4):821-30. https://doi.org/10.1242/dev.127.4 821.

26. Kelley R, Ren R, Pi X, Wu Y, Moreno I, Willis M, et al. A concentrationdependent endocytic trap and sink mechanism converts Bmper from an activator to an inhibitor of Bmp signaling. J Cell Biol. 2009;184(4):597-609. https://doi.org/10.1083/jcb.200808064.

27. Cao H, Jheon A, Li X, Sun Z, Wang J, Florez S, et al. The Pitx2:miR-200C/141: noggin pathway regulates Bmp signaling and ameloblast differentiation. Development. 2013;140(16):3348-59. https://doi.org/10.1242/dev.089193.

28. Esser JS, Rahner S, Deckler M, Bode C, Patterson C, Moser M. Fibroblast growth factor signaling pathway in endothelial cells is activated by BMPER to promote angiogenesis. Arterioscler Thromb Vasc Biol. 2015;35(2):358-67. https://doi.org/10.1161/ATVBAHA.114.304345.

29. Ishizaki H, Westermark A, van Setten G, Pyykkö I. Basic fibroblast growth factor (bFGF) in saliva--physiological and clinical implications. Acta Otolaryngol Suppl. 2000;543:193-5. https://doi.org/10.1080/0001648004543 78.

30. Fujihara C, Kanai Y, Masumoto R, Kitagaki J, Matsumoto M, Yamada S, et al. Fibroblast growth factor-2 inhibits CD40-mediated periodontal inflammation. J Cell Physiol. 2019;234(5):7149-60. https://doi.org/10.1002/ jcp.27469.

31. Lallier TE, Spencer A. Use of microarrays to find novel regulators of periodontal ligament fibroblast differentiation. Cell Tissue Res. 2007;327(1): 93-109. https://doi.org/10.1007/s00441-006-0282-5.

32. He X, Chao Y, Zhou G, Chen Y. Fibroblast growth factor 5-short (FGF5s) inhibits the activity of FGF5 in primary and secondary hair follicle dermal papilla cells of cashmere goats. Gene. 2016;575(2 Pt 2):393-8. https://doi. org/10.1016/j.gene.2015.09.034.

33. Eguchi K, Akiba Y, Akiba N, Nagasawa M, Cooper LF, Uoshima K. Insulin-like growth factor binding Protein-3 suppresses osteoblast differentiation via bone morphogenetic protein-2. Biochem Biophys Res Commun. 2018; 507(1-4):465-70. https://doi.org/10.1016/j.bbrc.2018.11.065.

34. Jones JI, Clemmons DR. Insulin-like growth factors and their binding proteins: biological actions. Endocr Rev. 1995;16(1):3-34. https://doi.org/1 0.1210/edrv-16-1-3.

35. Kawai M, Rosen CJ. The insulin-like growth factor system in bone: basic and clinical implications. Endocrinol Metab Clin North Am. 2012;41(2):323-33, vi. https://doi.org/10.1016/j.ecl.2012.04.013.

36. Saggese R, Federico G, Gandini P. The IGF-1--IGFBPs system in the crevicular fluid: its changes during orthodontic movement. Prog Orthod. 2005;6(1): 114-8 Available from: http://europepmc.org/abstract/MED/15977342.

37. Jia S, Zhou J, Gao Y, Baek J-A, Martin JF, Lan Y, et al. Roles of Bmp4 during tooth morphogenesis and sequential tooth formation. Development. 2013; 140(2):423-32. https://doi.org/10.1242/dev.081927.

38. Kim J-Y, Cho S-W, Hwang H-J, Lee M-J, Lee J-M, Cai J, et al. Evidence for expansion-based temporal BMP4/NOGGIN interactions in specifying periodontium morphogenesis. Cell Tissue Res. 2007;330(1):123-32. https:// doi.org/10.1007/s00441-007-0434-2.

39. Ou M, Zhao Y, Zhang F, Huang X. Bmp2 and Bmp4 accelerate alveolar bone development. Connect Tissue Res. 2015;56(3):204-11. https://doi.org/10.31 09/03008207.2014.996701.

40. Nakase T, Nomura S, Yoshikawa H, Hashimoto J, Hirota S, Kitamura Y, et al. Transient and localized expression of bone morphogenetic protein 4 messenger RNA during fracture healing. J Bone Miner Res Off J Am Soc Bone Miner Res. 1994;9(5):651-9.

41. Yu M, Wang H, Fan Z, Xie C, Liu H, Liu Y, et al. BMP4 mutations in tooth agenesis and low bone mass. Arch Oral Biol. 2019;103:40-6 Available from: https://europepmc.org/articles/PMC6639811.

42. Gluhak-Heinrich J, Guo D, Yang W, Harris MA, Lichtler A, Kream B, et al. New roles and mechanism of action of BMP4 in postnatal tooth cytodifferentiation. Bone. 2010;46(6):1533-45. https://doi.org/10.1016/j. bone.2010.02.024.

43. Ye L, Zhang S, Ke H, Bonewald L, Feng J. Periodontal breakdown in the Dmp1 Null mouse model of hypophosphatemic rickets. J Dent Res. 2008; 87(7):624-9. https://doi.org/10.1177/154405910808700708.

44. Huang H-Y, Hu L-L, Song T-J, Li X, He Q, Sun X, et al. Involvement of cytoskeleton-associated proteins in the commitment of C3H10T1/2 pluripotent stem cells to adipocyte lineage induced by BMP2/4. Mol Cell Proteomics. 2011;10(1):M110.002691. https://doi.org/10.1074/mcp.M110.002 691.

45. Li Q, Zhang S, Sui Y, Fu X, Li Y, Wei S. Sequential stimulation with different concentrations of BMP4 promotes the differentiation of human embryonic stem cells into dental epithelium with potential for tooth formation. Stem Cell Res Ther. 2019;10(1):276. https://doi.org/10.1186/s13287-019-1378-7.

46. Swietlik JJ, Sinha A, Meissner F. Dissecting intercellular signaling with mass spectrometry-based proteomics. Curr Opin Cell Biol. 2020;63:20-30. https:// doi.org/10.1016/j.ceb.2019.12.002

\section{Publisher's Note}

Springer Nature remains neutral with regard to jurisdictional claims in published maps and institutional affiliations.

\section{Submit your manuscript to a SpringerOpen ${ }^{\circ}$ journal and benefit from:}

- Convenient online submission

- Rigorous peer review

- Open access: articles freely available online

- High visibility within the field

- Retaining the copyright to your article

Submit your next manuscript at $>$ springeropen.com 